\title{
Statistical versus clinical significance
}

\author{
Bahaaldin Alsoufi, MD
}

From the Division of Cardiothoracic Surgery, Emory University School of Medicine, Children's Healthcare of Atlanta, Atlanta, Ga.

Disclosures: Author has nothing to disclose with regard to commercial support.

Received for publication Aug 27, 2017; accepted for publication Aug 30, 2017; available ahead of print Sept 21, 2017.

Address for reprints: Bahaaldin Alsoufi, MD, Division of Cardiothoracic Surgery, Emory University School of Medicine, Children's Healthcare of Atlanta, 1405 Clifton Rd, NE, Atlanta, GA 30322 (E-mail: balsoufi@ hotmail.com).

J Thorac Cardiovasc Surg 2018;155:344-5

$0022-5223 / \$ 36.00$

Copyright $\subset 2017$ by The American Association for Thoracic Surgery

http://dx.doi.org/10.1016/j.jtcvs.2017.08.108

Talwar and colleagues ${ }^{1}$ performed a prospectively randomized study where they divided a cohort of 50 children with tetralogy of Fallot (TOF) into 2 equal groups (25 patients each) who received either 5 doses of oral allopurinol $(10 \mathrm{mg} / \mathrm{kg} /$ dose $)$ or placebo at 12 hours before surgery; before moving to the operating room; and then at 12, 24, and 36 hours after surgery. They found that the postoperative levels of interleukin $1-\beta$, interleukin 6 , malondialdehyde, and plasma troponin-I were all statistically higher in the placebo group, suggesting that the use of allopurinol, a xanthine oxidase inhibitor, is associated with diminished inflammatory response and decreased myocardial cell injury following cardiopulmonary bypass (CPB) and ischemic cardiac arrest during TOF repair. At the same time, the authors noted that allopurinol use was associated with lower inotropic score (median, 10 vs $15 ; P=.02$ ), shorter mechanical ventilation (median, 7 vs 8 hours; $P=.01$ ), intensive care unit stay (median, 32 vs 48 hours; $P=.001$ ), and hospital stay (median, 5 vs 6 days; $P=.01$ ). The calculated cardiac index at 6 hours after surgery was higher in the allopurinol group, whereas the incidence of cardiac arrhythmias and the amount of pleural tube drainage were comparable. They concluded that the use of allopurinol might be associated with improved recovery and early outcomes following TOF repair.

The association between CPB and systemic inflammatory response is well established, although the full extent of this very complex process has not been entirely interpreted. $^{2-4}$ Similarly, the relationship between the activation of various inflammatory cascades during CPB and postoperative myocardial and end organ dysfunction with subsequent postoperative morbidity and prolonged recovery is well demonstrated, especially in the small neonates undergoing complex cardiac reconstructions. ${ }^{2-4}$ Naturally, this undesirable affiliation has prompted many investigators to explore various methods to decrease the inflammatory response to CPB. Those methods included alterations of the CPB circuit (eg, using biocompatible coated surfaces and miniaturization of the circuit), blood-

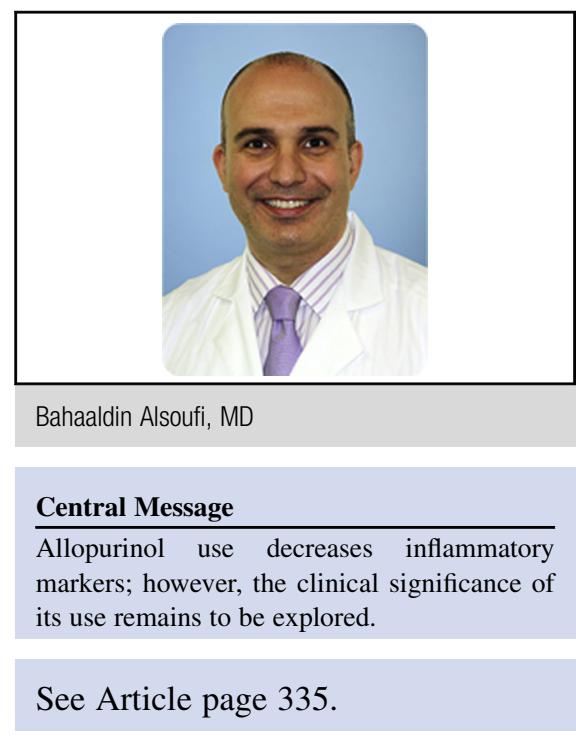

conservation methods, ultrafiltration, and the use of multiple anti-inflammatory agents such as aprotinin, corticosteroids, and aspirin to list a few. ${ }^{5,6}$ Corticosteroids are the most commonly studied medications that have been used as prophylactic anti-inflammatory therapy in children undergoing $\mathrm{CPB}$, although the type, timing, frequency, and dosage have widely varied among investigators. ${ }^{5-10}$ The results of corticosteroid use have been similarly diverse and whereas several studies showed reduction in inflammatory markers, the effects on postoperative hemodynamic parameters, recovery, and early clinical outcomes have not been consistent and in fact, a number of reports raised concern about the safety of corticosteroids and potential increased risk of infection, renal damage, and neurologic insult. ${ }^{5-10}$ Despite the large amount of research on the topic, the prophylactic preoperative and postoperative use of corticosteroids in congenital cardiac surgery remains debatable.

In this prospectively randomized study of the effect of allopurinol in modulating inflammatory response and myocardial dysfunction following CPB, the authors showed statistical differences in a number of the inflammatory markers and clinical parameters. ${ }^{1}$ The implications of this study on clinical practice are questionable. As we have learned from the numerous corticosteroid studies, the differences of postoperative inflammatory markers do not always correlate with differences in clinical outcomes. Additionally, the study population is not necessarily one that continues to be highly problematic in the current era of advanced perioperative care; operative mortality is very rare in nonneonatal TOF repair and postoperative recovery 
is commonly straightforward, aside from an occasional incidence of postoperative tachyarrhythmia. ${ }^{11}$ Inotropic score is generally used to compare the amount of inotropic and vasoactive agents used following cardiac surgery. Nonetheless, the use of those agents is not necessarily always guided by patient need but rather by what a physician anticipates a patient will need. There are surely variations among physicians and certainly between institutions in the threshold to initiate, the type, and the amount of inotropes and vasoactive agents, and in the timing and rate of weaning of those medications following surgery. In most institutions, the inotropic score following an elective TOF repair rarely exceeds 10 , which is similar to the score in patients who received allopurinol in the current study. Despite the statistical significance in inotropic score between the 2 study arms, it is difficult to demonstrate whether or not the amount and longevity of the use of those agents was dictated by the hemodynamic condition or clinical need of the patients. Additionally, whereas the duration of mechanical ventilation, intensive care unit stay, and hospital stays between the 2 groups of patients were all statistically lower in the allopurinol group (eg, a duration of ventilation 7 vs 8 hours), it remains questionable whether those are clinically significant, truly guided by patient needs and not influenced by logistic factors. For example, many programs implement a fast-track protocol following TOF repair with those patients extubated in the operating room, underscoring the physician factor that influences the recovery of those patients. ${ }^{12}$ The 2 reasons that commonly require prolonged intensive care unit or hospital stays following TOF repair are arrhythmias and prolonged drainage from the chest drains, and those were both comparable between the 2 groups of patients.

Rather than dismiss the findings of this nice, prospectively randomized study, ${ }^{1}$ we should consider where to proceed from here. Should we examine the effect of allopurinol on modifying the inflammatory response to CPB in vulnerable populations such as neonates undergoing complex cardiac procedures? Given the multitude of variables that influence the activation of those complex inflammatory cascades, would we be we able to perform single-institution studies powered to demonstrate allopurinol's effect on inflammatory markers and more importantly clinical parameters? Given that allopurinol is a cheap medication, it is unlikely that the industry will invest in a multiinstitutional effort to examine the role of this medication in this area. Should there be a multi-institutional effort to examine this medication? How can we avoid the many hurdles that have complicated similar studies examining corticosteroids in the past? What do we know about the safety of allopurinol use in small children undergoing CPB? Most of what we know about the side effects of this medication comes from chronic use in adults with other medical conditions. Despite about 2 decades of investigation into the role of corticosteroids in children undergoing $\mathrm{CPB}$, the issue remains debatable due to many of those limitations and it is likely that efforts examining the role of allopurinol in those patients will face the same limitations. Meanwhile, the statistical significance that has been demonstrated in this study might not be meaningful enough to influence a practical application.

\section{References}

1. Talwar S, Selvam MS, Makhija N, Lakshmy R, Choudhary S, Sreenivas V, et al. Effect of administration of allopurinol on postoperative outcomes in patients undergoing intracardiac repair of tetralogy of Fallot. J Thorac Cardiovasc Surg. 2018; 155:335-43

2. Stocker CF, Shekerdemian LS, Visvanathan K, Skinner N, Brizard CP, Carlin JB, et al. Cardiopulmonary bypass elicits a prominent innate immune response in children with congenital heart disease. J Thorac Cardiovasc Surg. 2004;127: 1523-5.

3. Mahle WT, Matthews E, Kanter KR, Kogon BE, Hamrick SE, Strickland MJ. Inflammatory response after neonatal cardiac surgery and its relationship to clinical outcomes. Ann Thorac Surg. 2014;97:950-6.

4. Holmes JH IV, Connolly NC, Paul DL, Hill ME, Guyton SW, Ziegler SF, et al. Magnitude of the inflammatory response to cardiopulmonary bypass and its relation to adverse clinical outcomes. Inflamm Res. 2002:51:579-86.

5. Shen I, Giacomuzzi C, Ungerleider RM. Current strategies for optimizing the use of cardiopulmonary bypass in neonates and infants. Ann Thorac Surg. 2003;75(2 Suppl):S729-34

6. Durandy Y. Minimizing systemic inflammation during cardiopulmonary bypass in the pediatric population. Artif Organs. 2014;38:11-8.

7. Scrascia G, Rotunno C, Guida P, Amorese L, Polieri D, Codazzi D, et al. Perioperative steroids administration in pediatric cardiac surgery: a meta-analysis of randomized controlled trials. Pediatr Crit Care Med. 2014;15:435-42.

8. Dreher M, Glatz AC, Kennedy A, Rosenthal T, Gaynor JW. A single-center analysis of methylprednisolone use during pediatric cardiopulmonary bypass. J Extra Corpor Technol. 2015;47:155-9.

9. Pasquali SK, Li JS, He X, Jacobs ML, O'Brien SM, Hall M, et al. Perioperative methylprednisolone and outcome in neonates undergoing heart surgery. Pediatrics. 2012;129:e385-91.

10. Graham EM, Atz AM, McHugh KE, Butts RJ, Baker NL, Stroud RE, et al. Preoperative steroid treatment does not improve markers of inflammation after cardiac surgery in neonates: results from a randomized trial. J Thorac Cardiovasc Surg. 2014;147:902-8.

11. Wilder TJ, Van Arsdell GS, Benson L, Pham-Hung E, Gritti M, Page A, et al Young infants with severe tetralogy of Fallot: rarely primary surgery versus transcatheter palliation. J Thorac Cardiovasc Surg. 2017;154:1692-700.e2.

12. Hamilton BC, Honjo O, Alghamdi AA, Caldarone CA, Schwartz SM, Van Arsdell GS, et al. Efficacy of evolving early-extubation strategy on early postoperative functional recovery in pediatric open-heart surgery: a matched casecontrol study. Semin Cardiothorac Vasc Anesth. 2014;18:290-6. 